\title{
Life cycle abbreviation in the trematode Coitocaecum parvum: can parasites adjust to variable conditions?
}

\author{
C. LAGRUE \& R. POULIN \\ Department of Zoology, University of Otago, Dunedin, New Zealand
}

\section{Keywords:}

abbreviated life cycle; alternative strategies;

Coitocaecum parvum;

phenotypic plasticity;

progenesis;

trematode.

\begin{abstract}
The complex life cycles of parasites are thought to have evolved from simple one-host cycles by incorporating new hosts. Nevertheless, complex developmental routes present parasites with a sequence of highly unlikely transmission events in order to complete their life cycles. Some trematodes like Coitocaecum parvum use facultative life cycle abbreviation to counter the odds of trophic transmission to the definitive host. Parasites adopting life cycle truncation possess the ability to reproduce within their intermediate host, using progenesis, without the need to reach the definitive host. Usually, both abbreviated and normal life cycles are observed in the same population of parasites. Here, we demonstrate experimentally that C. parvum can modulate its development in its amphipod intermediate host and adopt either the abbreviated or the normal life cycle depending on current transmission opportunities or the degree of intra-host competition among individual parasites. In the presence of cues from its predatory definitive host, the parasite is significantly less likely to adopt progenesis than in the absence of such cues. An intermediate response is obtained when the parasites are exposed to cues from non-host predators. The adoption of progenesis is less likely, however, when two parasites share the resource-limited intermediate host. These results show that parasites with complex developmental routes have transmission strategies and perception abilities that are more sophisticated than previously thought.
\end{abstract}

\section{Introduction}

Life cycles involving several host species are observed in numerous and phylogenetically distant groups of parasites (Poulin, 1998). Among those, the complex life cycles of parasitic helminths are thought to have evolved from simple one-host cycles by incorporation of new hosts (Parker et al., 2003a). For example, a predator might have been added to a parasite life cycle if it frequently consumed prey used by a parasite as host, therefore becoming a definitive host. In the case of trematodes, the ancestral single host was a vertebrate and new intermediate hosts were added during the course of evolution (Cribb et al., 2003). First, adopting a vertebrate as a host may have allowed high reproduction rates in hosts of

Correspondence: Clément Lagrue, Department of Zoology, University of Otago, PO Box 56, Dunedin 9054, New Zealand.

Tel.: +64 3479 7964; fax: +64 3479 7584;

e-mail: lagc1981@student.otago.ac.nz large body size and long life-span (Parker et al., 2003a), and a greater rate of cross-fertilization by concentrating isolated individuals (Brown et al., 2001). Then, the incorporation of intermediate hosts, used as transmission vectors, might have increased the probability of reaching a new vertebrate host individual (Choisy et al., 2003). Trematodes are also known to undergo active asexual multiplication within their mollusk first host, thus increasing their likelihood of transmission (Cribb et al., 2003). Nevertheless, complex transmission routes present parasites with a succession of highly hazardous transmission events in order to complete their life cycle, reach maturity and reproduce. This is particularly true in trophic transmission when the infected intermediate host must be eaten by the definitive host for the parasite to achieve its maximum growth and reproduce. It is well known that parasites relying on trophic transmission often alter the behaviour or appearance of their intermediate host to increase the likelihood of predation by the predator host (Moore, 2002). In several groups, some 
parasites use more extreme adaptations to counter the odds of trophic transmission: among trematodes, for instance, several species are able to drop the definitive host from their life cycle (Poulin \& Cribb, 2002). Those parasites produce eggs while still in their intermediate host via progenesis; the worms achieve precocious maturity and lay viable eggs by self-fertilization (Poulin, 2001). Although some species display extreme forms of life cycle truncation with only one host remaining, most life cycle truncations are facultative with both strategies present in the population. Conditions that trigger the adoption of one or the other strategy by parasites are currently poorly understood.

Poulin (2003) demonstrated that a trematode parasite can accelerate its development and reach precocious maturity in its intermediate host in the absence of definitive hosts. This result illustrates that some parasites may adjust their life strategy with respect to their immediate transmission opportunities to an appropriate definitive host. However, in a wider ecological context, other predators unsuitable as hosts may actively feed on the same prey species and significantly influence the benefit of one or the other strategy (Mouritsen $\delta$ Poulin, 2003). Thomas et al. (2002a) suggested that selection may have favoured parasites with the ability to perceive a diversity of signals from environmental parameters affecting their immediate and future fitness. Thus, parasites might be able to strategically respond to a diversity of signals emanating from their host environment such as the presence of predators (Combes et al., 2002). Crustaceans are often used as intermediate hosts by helminth parasites; in addition, crustaceans are known to perceive the presence of fish predators by detecting the chemical components of fish mucus in the water (e.g. Lehtiniemi \& Lindén, 2006) and change their behaviour accordingly (Neill, 1990; Burks et al., 2000). Consequently, parasites needing either to avoid predation by a fish or to finish their life cycle in a fish gut should adopt different strategies based on their detection of predator-induced stress in the intermediate host. However, the actual perception ability of parasites and how effectively they can adjust their life strategies is currently unknown.

The present study addresses this important question using the same model as Poulin (2003). The trematode Coitocaecum parvum (Opecoelidae) is a common parasite of freshwater fish in New Zealand (MacFarlane, 1939; Holton, 1984a), principally the common bully (Gobiomorphus cotidianus). Eggs are released in fish faeces and hatch into free-swimming larvae (miracidia). Miracidia penetrate snails in which they multiply and develop into sporocysts. Sporocysts asexually produce cercariae, freeliving larvae that enter the amphipod Paracalliope fluviatilis where they encyst as metacercariae in the body cavity. At this stage, metacercariae can either stop growing and await ingestion by a fish where they will mature and reproduce, or keep growing and reach maturity while still inside the amphipod. Worms that reach maturity in the crustacean intermediate host reproduce by selfing and lay eggs in the amphipod's body cavity (Holton, 1984b; Poulin, 2001). Eggs produced by selfing hatch after host death into larvae that are infective to the snail first host without the need to pass through the fish host. Coitocaecum parvum can adopt preferentially the shorter life cycle in the absence of chemical cues emanating from its preferred fish definitive host, the common bully (Poulin, 2003). However, Poulin (2003) used naturally infected amphipods, and it is possible that variation in the age of metacercariae may have confounded the results. It is also important to determine if this parasite is able to distinguish between its fish definitive host and other nonhost predators and adapt its life strategy accordingly as multiple nonhost predators commonly co-occur with G. cotidianus.

Here, we tested the hypothesis, using experimental infections, that the trematode C. parvum can use information about current opportunities of transmission to its fish definitive host to adjust its life-history strategy. We expect that the parasite will shorten its life cycle not only when the opportunities for transmission to the definitive host are limited (low density of hosts), but also when other predators not suitable as definitive hosts are present, significantly decreasing the probability of transmission to the appropriate host. In these two different situations, the parasite may use progenesis to guarantee the production of at least a few eggs before its amphipod host dies or is eaten by a nonhost predator. However, the proportion of worms adopting an abbreviated life cycle and the rate of egg production might be different between situations where there is no predator and situations where nonhost predators are present. The presence of any predator, even if not suitable as a host for C. parvum, may induce stress in the amphipod that is perceived by the parasite and that influences its lifestrategy.

\section{Materials and methods}

\section{Animal collection}

Naturally infected snails (Potamopyrgus antipodarum) and amphipods (Paracalliope fluviatilis) were collected among macrophytes in Lake Waihola, South Island, New Zealand using dip nets. Snails and amphipods were kept alive in aerated lake water before the experiments and stocks of both animals were renewed every fortnight to control for the delay before processing. Infected snails were obtained by selectively choosing individuals that displayed an altered shell shape, a sure sign of infection by C. parvum (C. Lagrue, personal observation). Uninfected amphipods were obtained by inspecting each amphipod under a microscope and discarding all amphipods that showed any sign of infection, i.e. an opaque mass in the body cavity corresponding to a metacercaria. 
This method allows the selection of only uninfected individuals with an accuracy of about 95\% (Lefebvre 8 Poulin, 2005a). Twenty-five common bullies, the preferred definitive host of C. parvum, and 25 perch (Perca fluviatilis), a nondefinitive host predator (Poulin, 2003), were collected in Lake Waihola using a set net, kept alive separately in $200 \mathrm{~L}$ tanks and fed with commercial fish food. Twenty-five crayfish (Paranephrops zealandicus), another nondefinitive host predator (Hollows et al., 2002), were captured by hand in Ross Creek, within Dunedin city, using a spot light after dark.

\section{Experimental infections}

Cercariae of the trematode parasite C. parvum were obtained from snails under controlled conditions to ensure that the cercariae used to experimentally infect amphipods were freshly released and, therefore, more likely to penetrate the amphipod. Randomly chosen snails were transferred to Petri-dishes (about 50 snails per dish) filled will $10 \mathrm{~mL}$ of filtrated lake water. Snails were then incubated at $25^{\circ} \mathrm{C}$ for 20 min under constant light, conditions known to induce cercarial release (Hay et al., 2005). The Petri-dishes were then screened under a microscope and the cercariae found were transferred to $500 \mu \mathrm{L}$ Eppendorf tubes using a $20 \mu \mathrm{L}$ micropipette. Two cercariae were placed in each tube with $2.5 \mu \mathrm{L}$ of filtrated lake water and an amphipod was then added. Amphipods were left in the tube along with the two cercariae for $5 \mathrm{~h}$, a time after which unsuccessful cercariae stop moving and die. Amphipod survival, at this stage, was over $99 \%$. Amphipods were then separated randomly into groups of about 50 individuals. Each group was placed in a plastic container filled with $400 \mathrm{~mL}$ of aged and aerated lake water; strands of macrophytes (Elodea canadensis) were added for food. Aged water consisted of water collected at least 1 week before the experiment to allow any chemical cue to deteriorate (Poulin, 2003). Given that 3414 amphipods were experimentally infected for the purpose of this study, controlled infections and treatments were conducted over several weeks between July and October 2005.

\section{Treatment}

All types of scented water were prepared every second day following the same protocol: $2.5 \mathrm{~L}$ of aged lake water were placed in each of four identical plastic tanks, and four individuals of either perch, common bully or crayfish, randomly chosen from a pool of 25 , were added separately to three of the four tanks. The fourth tank was left with only water (control water) to standardize the treatment. Animals were left overnight and removed in the morning. The different types of treated water were immediately used and added to the tanks containing the experimentally infected amphipods. Every 2 days and for 5 weeks, $60 \mathrm{~mL}$ of water was removed from each amphipod container and replaced with either control water or water conditioned by one of the three predators. The same treatment was applied to a given container for the whole experiment. In total, 730 amphipods served as control, 1190 received bully water, 702 received perch water and 792 were treated with crayfish-marked water.

\section{Measures and statistical analyses}

After 5 weeks, all surviving amphipods $(309,388,80$ and 277 respectively for each treatment) were killed in $70 \%$ ethanol to facilitate dissections and measurements, rinsed in distilled water and dissected immediately after. Amphipods were measured (body length) and dissected under the microscope. Any worm they contained was measured (length and width) under a compound microscope, and recorded as 'normal' (nonegg producing worm) or 'progenetic' (egg producing worm); in the case of progenetic parasites, eggs were also counted. The body surface of each parasite was then determined and used as a surrogate for body size. This was done using the formula for an ellipsoid, $(\pi L W) / 4$, where $L$ and $W$ are the length and width of the parasite. As amphipods were experimentally exposed to two cercariae, some individuals contained two metacercariae of C. parvum, whereas others had only one or were uninfected. Because competition between individuals sharing the same host is an important factor influencing life-history strategies and growth of parasites (Thomas et al., 2002b; Parker et al., 2003b), recovered worms were divided in two different classes under 'infection status': single infections (one worm per amphipod) and double infections (two worms per amphipod).

Linear regressions were applied to test for the effect of amphipod size on the size of the parasites. Effects of treatment and infection status (single or double infection) on parasite strategy were tested using Fisher's exact tests; the proportions of parasites in each class were compared in a pair-wise manner. Effects of treatment, infection status and strategy ('normal' or 'progenetic') on parasite body size were tested using a three-way Anova with the size of the worm used as the dependent variable. The body area of the parasite was log transformed before analyses to normalize the data. The effect of the treatment on egg production was tested using a nonparametric test (Kruskal-Wallis Anova) with the number of eggs used as the dependent variable and the type of treatment as the independent variable; only parasites that had produced at least one egg were included in this analysis. Finally, a linear regression between the size of the parasite and the number of eggs produced was used to assess the effect of parasite size on egg production.

\section{Results}

Overall, 629 metacercariae of C. parvum were recovered from the 1054 surviving amphipods. Of these 1054 
individuals, 486 had been successfully infected: 343 had one metacercaria and 143 had two. However, because it was impossible to know the proportion of dead amphipods that was parasitized, we could not determine the actual percentage of individuals infected at the beginning of the experiment.

Amphipod length did not differ among treatments (F3, $482=1.39, P=0.24)$ and, thus, all infected amphipods were used to test for the effect of amphipod size on the size of the parasites. Across all treatments, the body size of normal worms was not related to amphipod length either in single infections $(r=0.035, n=222, P=0.61)$ or in double infections $(r=0.093, n=245, P=0.15)$; there were also no significant correlations between these variables within any of the treatments. On the contrary, the body size of progenetic parasites found in single infections was significantly related to host length $(r=$ $0.19, n=121, P<0.05)$. This weak trend was also observed in progenetic worms found in double infections but was not significant probably because of the smaller sample size $(r=0.17, n=41, P=0.29)$.

In single infections (one parasite per host), the proportion of progenetic worms in the bully treatment was significantly lower than in any other treatment (Table 1A and Fig. 1). The control treatment showed the significantly highest percentage of progenetic parasites while there was no difference between the perch and crayfish treatments (Table 1A and Fig. 1). In double infections, those differences in occurrence of progenesis were less pronounced (Table 1B) and, apart from the bully treatment, the percentage of progenetic parasites was clearly lower than in single infections (Table 2 and Fig. 1). However, the difference was not significant in the perch treatment, probably due to the small sample size.

The growth achieved by the parasites was significantly different among the four treatments (Table 3): worms

Table 1 Results of Fisher's exact tests for pair-wise comparisons of the proportion of progenetic parasites between treatments: (A) for parasites in single infections and (B) for parasites in double infections.

\begin{tabular}{lrr}
\hline Treatments compared & \multicolumn{1}{c}{$\chi^{2}$} & $P$-value \\
\hline A & 124.67 & $<0.0001$ \\
Control vs. bully & 19.37 & $<0.0001$ \\
Control vs. crayfish & 17.35 & 0.0001 \\
Control vs. perch & 51.66 & $<0.0001$ \\
Bully vs. crayfish & 21.87 & 0.0001 \\
Bully vs. perch & 0.66 & 0.276 \\
Crayfish vs. perch & & 0.045 \\
B & 4.03 & 0.367 \\
Control vs. bully & 0.32 & 0.627 \\
Control vs. crayfish & 0.00 & 0.003 \\
Control vs. perch & 8.65 & 0.160 \\
Bully vs. crayfish & 2.10 & 0.501 \\
Bully vs. perch & 0.13 & \\
Crayfish vs. perch & & \\
\hline
\end{tabular}

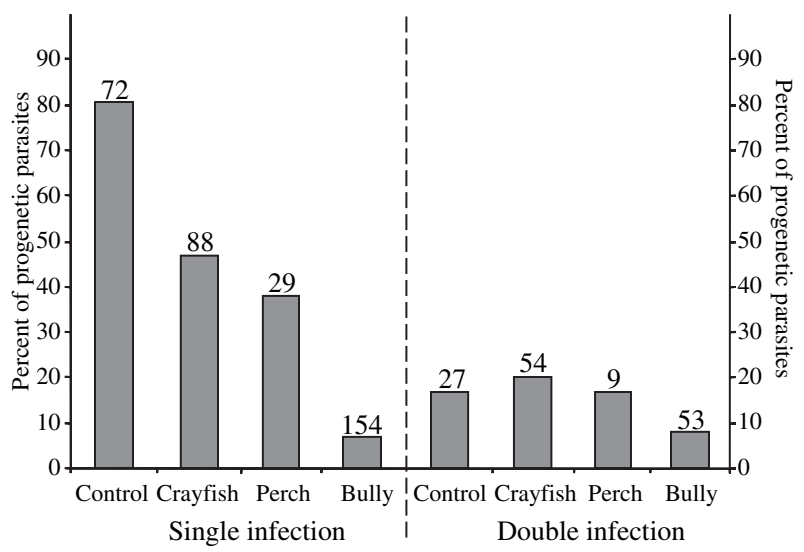

Fig. 1 Proportion of egg producing (i.e. progenetic) parasites in each treatment as a function of their infection status (i.e. single or double infection). Numbers above bars are the number of infected amphipods in each category.

Table 2 Results of Fisher's exact tests for comparisons of the proportion of progenetic parasites between single and double infections within each treatment.

\begin{tabular}{lcc}
\hline Single vs. double & $\chi^{2}$ & $P$-value \\
\hline Control & 50.58 & $<0.0001$ \\
Bully & 0.03 & 0.537 \\
Crayfish & 15.28 & 0.0001 \\
Perch & 2.40 & 0.110 \\
\hline
\end{tabular}

Table 3 Results of the three-way Anova using treatment, infection status and life-history strategy as independent variables and the size of the parasites as the dependent variable.

\begin{tabular}{llrc}
\hline Main effects & d.f. & \multicolumn{1}{l}{$F$} & $P$-value \\
\hline Treatment & 3 & 5.543 & 0.0009 \\
Infection status & 1 & 2.813 & 0.094 \\
Life-history strategy & 1 & 568.723 & $<0.0001$ \\
Treatment $\times$ infection status & 3 & 1.147 & 0.329 \\
Treatment $\times$ strategy & 3 & 2.859 & 0.036 \\
Infection status $\times$ strategy & 1 & 10.405 & 0.0013 \\
Treatment $\times$ infection status $\times$ strategy & 3 & 0.310 & 0.818 \\
\hline
\end{tabular}

exposed to control water were significantly larger $\left(0.125 \pm 0.007 \mathrm{~mm}^{2}\right)$ than in the bully $\left(0.04 \pm 0.003 \mathrm{~mm}^{2}\right)$, crayfish $\left(0.081 \pm 0.005 \mathrm{~mm}^{2}\right)$ and perch $\left(0.095 \pm 0.01 \mathrm{~mm}^{2}\right)$ treatments (Fisher's LSD, d.f. $=613$, all $P<0.001$ ), while trematodes from the bully treatment were the smallest (Fisher's LSD, d.f. $=$ 613, all $P<0.001)$. The life-history strategy also proved to have an effect on parasite mean body size: progenetic parasites were significantly larger than normal ones (Table 3). In contrast, the infection status (single or double) had no significant effect on trematode size. However, there was a significant interaction between the 


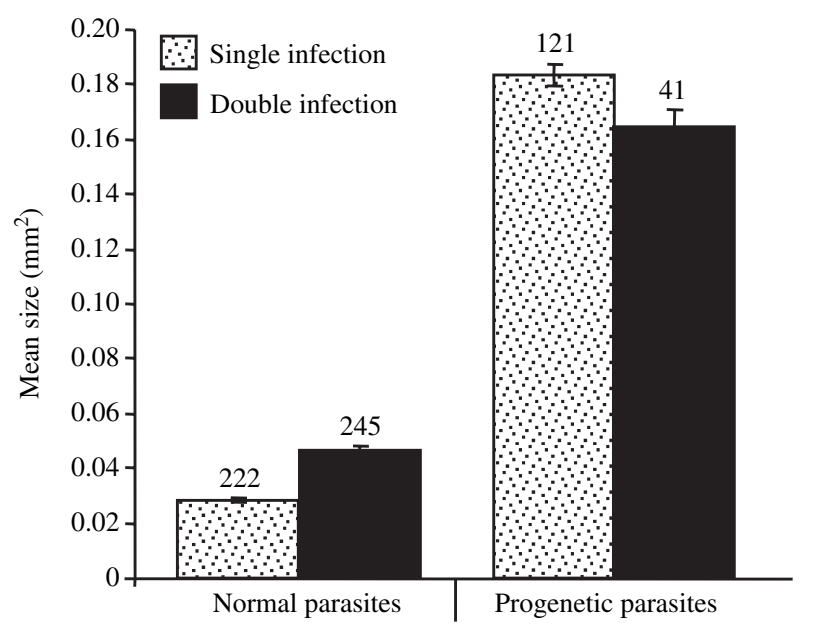

Fig. 2 Effects of life-history strategy and infection status on the mean size $( \pm \mathrm{SE}$ ) of parasites (see Table 3 for results of Anova). Post hoc tests showed a significant influence of the infection status on the size of normal parasites (Fisher's LSD, d.f. $=613, P<0.0001$ ) while no significant difference was found in progenetic parasites between single and double infections (Fisher's LSD, d.f. $=613, P=0.191$ ). Numbers above bars are sample sizes.

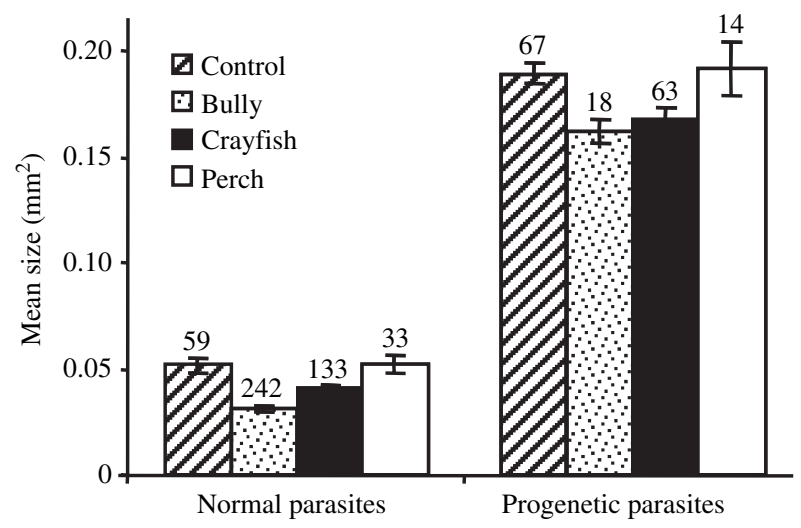

Fig. 3 Effects of treatment and life-history strategy on the mean size $( \pm$ SE) of parasites (see Table 3 for results of Anova). Post hoc tests showed significant effects of the different types of treatment on the size of normal parasites: worms in the bully treatment were significantly smaller than in any other treatment (Fisher's LSD, d.f. $=613$, all $P<0.0001$ ) while parasites exposed to control water were significantly larger than those in crayfish treatment but not in perch treatment (Fisher's LSD, d.f. $=613, P<0.0001$ and $P=0.67$ respectively). No significant effect was detected within progenetic parasites (Fisher's LSD, d.f. $=613$, all $P>0.13$ ).

life-history strategy and the infection status (see Table 3 and Fig. 2): normal parasites were significantly larger when found in double infections than in single infections while there was no significant difference among progenetic individuals. There was also a significant interaction between the life-history strategy and the type of treatment (see Table 3 and Fig. 3): the mean size of

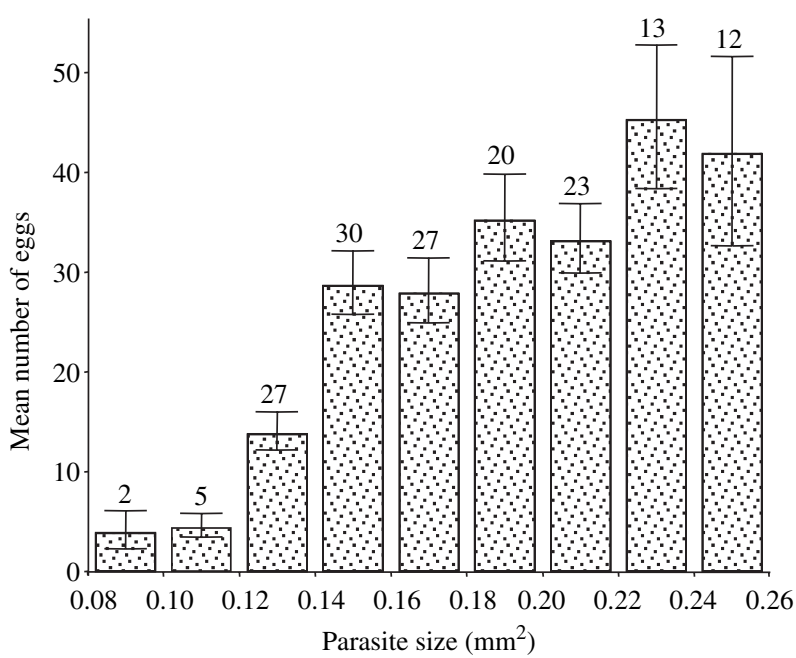

Fig. 4 Relationship between the mean $( \pm S E$ ) number of eggs produced and the size of the parasite. Only progenetic worms were used for this analysis. Numbers above bars are sample sizes.

progenetic parasites was less affected by the treatment than that of normal parasites. Finally, there were no other significant interactions (Table 3 ).

The mean number of eggs produced per progenetic parasite $\quad(27.4 \pm 2.6, \quad 19.7 \pm 2.4, \quad 23.3 \pm 2.4 \quad$ and $28.6 \pm 6.1$ for Control, bully, crayfish and perch treatments respectively) was not influenced by the type of treatment (Kruskal-Wallis ANovA, H3, $162=2.273$, $P=0.517)$ but increased significantly with the size of the parasite $(r=0.581, n=162, P<0.0001$; Fig. 4$)$.

\section{Discussion}

The likelihood of completing all transmission events is considered as the main force acting on the evolution of abbreviated life cycles (Poulin \& Cribb, 2002). Poulin (2001) suggested that the high density of amphipods and the presence in New Zealand freshwaters of other crustacean and fish predators, not suitable as definitive hosts, induce an extremely low probability of transmission to the definitive host for C. parvum. Therefore, hazardous transmission events may have promoted the evolution of progenesis as an alternative strategy, giving the trematode a wider range of transmission options. Coitocaecum parvum should then be able to adjust its developmental strategy to its immediate transmission opportunities. The present study shows that this trematode not only adopts preferentially progenesis in the absence of chemical cues from common bullies (G. cotidianus), its definitive host (as found by Poulin, 2003), but a high proportion of individual parasites grow larger and produce eggs within amphipods exposed to chemical cues emanating from other nonhost predators, the perch (Perca fluviatilis) and the crayfish (Paranephrops zealandicus). In other words, the developmental strategy 
of C. parvum is conditional upon both opportunities for transmission and furthermore upon the risk of 'dead-end transmission'.

Whereas crustaceans are known to respond rapidly and specifically to very different types of chemical or physical stimulations (Boersma et al., 1998; Burks et al., 2000; Meerhoff et al., 2006), C. parvum must detect physiological changes in its amphipod host associated with specifically induced responses that are relevant to its transmission opportunities. The parasite then responds by either interrupting its growth when the physiological level of stress within the host translates into the presence of the definitive host predator in the surroundings, or by adopting progenesis when no predator-related physiological response occurs in the amphipod. Here, we clearly demonstrate that $C$. parvum is able to adjust its life cycle to the presence or absence of its definitive host. However, although the total absence of predator or the presence of the historically preferred host trigger strong and advantageous responses from the parasite, the presence of other predatory nondefinitive hosts produce less clearcut responses by the trematode $C$. parvum. The intermediate situation observed when infected amphipods are exposed to nonhost predator odours could be explained in two different ways. First, in addition to being the historical definitive host for C. parvum, the common bully is the more abundant fish in many New Zealand waterways and feeds mostly on small invertebrates (McDowall, 1990). Consequently, amphipods may react strongly to chemical signals from this particular fish and display more intense physiological changes than when exposed to other less dangerous or rarer predators. In this scenario, parasitic worms only respond to different intensities of the same physiological changes with no efficient way of differentiating between types of predators, therefore making the best of a bad situation. Secondly, different types of predators may produce different types of chemicals released in the water. If amphipods are capable of detecting those different chemicals and recognizing the type of predator such that they respond differently in terms of behaviour and also physiologically, then this would open a small window on the outside world to the parasite. However, because the parasite detects those cues through its host, its perception must not be as accurate as that of the host itself. The fact that perch (Perca fluviatilis) has been introduced recently in New Zealand waters (McDowall, 1990) may also explain why parasites are less capable of correctly adjusting their strategy when the amphipod host is exposed to perch scent. The simple fact that we can measure a response of the parasite tends to prove that both amphipods and their parasites have developed the ability, to a certain extent, to detect and react to the presence of a completely new predator phylogenetically distant from the common bully. As a whole, this is the first experimental study showing that some parasites are capable of specific state dependent responses to several different stimuli, as suggested by Thomas et al. (2002a). Nevertheless, whether parasites respond to different intensities of the same signal or are capable of detecting several different stimuli remains to be investigated.

The external environment exerts a strong influence on the life history of internal parasites and induces changes in state dependent strategies adopted by these parasites (Poulin \& Cribb, 2002). Another source of environmental heterogeneity is the competition between individual parasites sharing the same intermediate host (Parker et al., 2003b). As parasites rely only on the limited space and energy available within the host, several individuals using the same host may be faced with resource constraints that could influence their development (Brown et al., 2003; Fredensborg \& Poulin, 2005). This is especially true with $C$. parvum where the size of progenetic worms is positively correlated with the size of the host. Thus, an individual adopting progenesis will use most if not all of the space and resources available in its host to grow fast and produce as many eggs as possible. There is an obvious area of conflict in cases where several worms have to share the same host. Accordingly, the results show a much lower proportion of progenetic worms among parasites found in double infections. This demonstrates that the life-history strategy of parasites is modulated by both the resource availability within the host and the final host availability associated with chemical cues from the external environment. However, the differences in sizes of normal parasites observed between single and double infections could mean that some worms in double infections are still growing and will eventually produce eggs. In this case, because the overall egg production is correlated with worm size, there will be a strong competition for space and energy if both worms adopt progenesis. Even if individuals adopt different strategies, each one may also have consequences and costs for the other individual parasite. Indeed, there is a conflict of interests between the two distinct life cycles that is potentially very costly for one of the two parasites (Dezfuli et al., 2001): intermediate host death for progenesis vs. ingestion by a definitive host fish for the classic 3-host route. Thus, if the host dies before being eaten by a fish, the normal worm will die along with its host without any offspring being produced. Genetic relatedness may greatly influence developmental strategies of parasites: closely related individuals should exploit the resources of a common host in a more cooperative and equitable manner (kin selection) than nonrelated parasites (Frank, 1992; Griffin \& West, 2002).

Altogether, our results show that growth rates and lifehistory strategies in the trematode C. parvum are even more plastic than previously thought (Poulin, 2003), proving that some parasitic organisms are capable of accurate adjustments of their life cycles. The two alternative developmental cycles used by the trematode C. parvum are likely to be a demonstration of adaptive phenotypic plasticity where the net fitness benefits of each 
strategy may vary depending on the transmission opportunities, thus allowing the maintenance of both strategies in the population. The intra-host competition between individual parasites may also play an important role in their life-history strategy, but whether genetic relatedness between co-occurring parasites influences their developmental strategy remains to be investigated. It will also be interesting to test whether the many other trematode species capable of facultative progenesis (Lefebvre $\delta$ Poulin, 2005b) are also capable of detecting opportunities for transmission and of responding accordingly.

\section{Acknowledgments}

We thank Devon Keeney for comments on an earlier version of the paper. This research was supported by the Marsden Fund (The Royal Society of New Zealand).

\section{References}

Boersma, M., Spaak, P. \& De Meester, L. 1998. Predatormediated plasticity in morphology, life history, and behavior of Daphnia: the uncoupling of responses. Am. Nat. 152: 237248.

Brown, S.P., Renaud, F., Guegan, J.F. \& Thomas, F. 2001. Evolution of trophic transmission in parasites: the need to reach a mating place? J. Evol. Biol. 14: 815-820.

Brown, S.P., De Lorgeril, J., Joly, C. \& Thomas, F. 2003. Field evidence for density-dependent effects in the trematode Microphallus papillorobustus in its manipulated host, Gammarus insensibilis. J. Parasitol. 89: 668-672.

Burks, R.L., Jeppesen, E. \& Lodge, D.M. 2000. Chemicals from macrophytes and fishes suppress Daphnia growth and alter life history traits. Oikos 88: 139-147.

Choisy, M., Brown, S.P., Lafferty, K.D. \& Thomas, F. 2003. Evolution of trophic transmission in parasites: why add intermediate hosts? Am. Nat. 162: 172-181.

Combes, C., Bartoli, P. \& Théron, A. 2002. Trematode transmission strategies. In: The Behavioural Ecology of Parasites (E. E. Lewis, J. F. Campbell \& M. V. K. Sukhdeo, eds), pp. 1-12. CAB International, Wallingford, UK.

Cribb, T.H., Bray, R.A., Olson, P.D. \& Littlewood, D.T.J. 2003. Life cycle evolution in the digenean: a new perspective from phylogeny. Adv. Parasitol. 54: 197-254.

Dezfuli, B.S., Giari, L. \& Poulin, R. 2001. Costs of intraspecific and interspecific host sharing in acanthocephalan cystacanths. Parasitology 122: 483-489.

Frank, S.A. 1992. A kin selection model for the evolution of virulence. Proc. R. Soc. Lond. B 250: 195-197.

Fredensborg, B.L. \& Poulin, R. 2005. Larval helminths in intermediate hosts: Does competition early in life determine the fitness of adult parasite? Int. J. Parasitol. 35: 1061-1070.

Griffin, A.S. \& West, S.A. 2002. Kin selection: fact and fiction. Trends Ecol. Evol. 17: 15-20.

Hay, K.B., Fredensborg, B.L. \& Poulin, R. 2005. Trematodeinduced alterations in shell shape of the mud snail Zeacumantus subcarinatus (Prosobranchia: Batillariidae). J. Mar. Biol. Ass. UK 85: 989-992.

Hollows, J.W., Townsend, C.R. \& Collier, K.J. 2002. Diet of the crayfish Paranephrops zealandicus in bush and pasture streams: insights from stable isotopes and stomach analysis. New Zealand J. Mar. Fresh. Res. 36: 129-142.

Holton, A.L. 1984a. A redescription of Coitocaecum parvum Crowcroft, 1945 (Digenea: Allocrediidae) from crustacean and fish hosts in Canterbury. New Zealand J. Zool. 11: 1-8.

Holton, A.L. 1984b. Progenesis as a mean of abbreviating life histories in two New Zealand trematodes, Coitocaecum parvum Crowfton, 1945 and Stegodexamene anguillae MacFarlane, 1951. Mauri Ora 11: 63-70.

Lefebvre, F. \& Poulin, R. 2005a. Alternative reproductive strategies in the progenetic trematode Coitocaecum parvum: comparison of selfing and mating worms. J. Parasitol. 91: 93-98.

Lefebvre, F. \& Poulin, R. 2005b. Progenesis in digenean trematodes: a taxonomic and synthetic overview of species reproducing in their second intermediate hosts. Parasitology 130: $1-19$.

Lehtiniemi, M. \& Lindén, E. 2006. Cercopagis pengoi and Mysis spp. Alter their feeding rate and prey selection under predation risk of herring (Clupea harengus membras). Mar. Biol. 149: 845-854.

MacFarlane, W.V. 1939. Life cycle of Coitocaecum anaspidis Hickman, a New Zealand digenetic trematode. Parasitology 31: 172-184.

McDowall, R.M. 1990. New Zealand Freshwater Fishes: A Natural History and Guide. MAF Publishing Group, Wellington.

Meerhoff, M., Fosalba, C., Bruzzone, C., Mazzeo, N., Noordven, W. \& Jeppesen, E. 2006. An experimental study of habitat choice by Daphnia: plants signal danger more than refuge in subtropical lakes. Fresh. Biol. 51: 1320-1330.

Moore, J. 2002. Parasites and the Behaviour of Animals. Oxford university press, Oxford.

Mouritsen, K.M. \& Poulin, R. 2003. Parasite-induced trophic facilitation exploited by a non-host predator: a manipulator's nightmare. Int. J. Parasitol. 33: 1043-1050.

Neill, W.E. 1990. Induced vertical migration in copepods as a defense against invertebrate predation. Nature 345: 524-526.

Parker, G.A., Chubb, J.C., Ball, M.A. \& Roberts, G.N. 2003 a. Evolution of complex life cycles in helminth parasites. Nature 425: 480-484.

Parker, G.A., Chubb, J.C., Roberts, G.N., Michaud, M. \& Milinski, M. 2003b. Optimal growth of larval helminths in their intermediate host. J. Evol. Biol. 16: 47-54.

Poulin, R. 1998. Evolutionary Ecology of Parasites: From Individuals to Communities. Chapman \& Hall, London.

Poulin, R. 2001. Progenesis and reduced virulence as an alternative transmission strategy in a parasitic trematode. Parasitology 123: 623-630.

Poulin, R. 2003. Information about transmission opportunities triggers a life-history switch in a parasite. Evolution 57: 28992903.

Poulin, R. \& Cribb, T.H. 2002. Trematode life cycles: short is sweet? Trends Parasitol 18: 176-183.

Thomas, F., Brown, S.P., Sukhdeo, M. \& Renaud, F. 2002a. Understanding parasite strategies: a state-dependent approach? Trends Parasitol. 18: 387-390.

Thomas, F., Fauchier, J. \& Lafferty, K.D. 2002b. Conflict of interest between a nematode and a trematode in an amphipod host: test of the 'sabotage' hypothesis. Behav. Ecol. Sociobiol. 51: 296-301.

Received 31 August 2006; revised 9 October 2006; accepted 13 October 2006 